\title{
Internal Limiting Membrane Inverted Flap Technique for Lamellar Macular Hole - Case Report
}

\author{
Florian Baltă ${ }^{1-3}$, Ioana Teodora Tofolean*2,3 and Ramona Barac ${ }^{1-3}$ \\ ${ }^{1}$ Clinical Emergency Eye Hospital, Romania \\ ${ }^{2}$ Carol Davila University of Medicine and Pharmacy, Romania \\ ${ }^{3}$ Retina Clinic, Ponderas Academic Hospital, Romania
}

*Corresponding author: Ioana Teodora Tofolean, Carol Knappe 63, 010508, Bucharest, Romania

\section{ARTICLE INFO}

Received: 幽 November 26, 2019

Published: 凿December 02, 2019

Citation: Florian Baltă, Ioana Teodora Tofolean, Ramona Barac. Internal Limiting Membrane Inverted Flap Technique for Lamellar Macular Hole - Case Report. Biomed J Sci \& Tech Res 23(3)-2019. BJSTR. MS.ID.003892.

Abbreviations: ILM: Internal Limiting Membrane; BE: Both Eyes; VA: Visual Acuity, RE: Right Eye; LE: Left Eye; OCT: Clinical and Optical Coherence Tomography; LMH: Lamellar Macular Hole; LHEP: Lamellar Hole Epiretinal Membrane; FTMH: Full Thickness Macular Hole

\section{ABSTRACT}

Objective: To describe one case of idiopathic lamellar macular hole associated with epiretinal membrane (ERM) surgically treated using the internal limiting membrane (ILM) inverted flap technique.

Methods: Interventional case report.

Results: The patient first presented to our clinic with both eyes (BE) 6/6 visual acuity (VA) two years before the current visit, when being diagnosed with BE epiretinal membrane without any tomographic sign of retinal layers' disruption. On the current examination, Snellen's VA was 6/6 in the right eye (RE) and 6/19 in the left eye (LE). Clinical and optical coherence tomography (OCT) appearances were compatible with the diagnosis of BE lamellar macular hole (LMH) and ERM. The patient's LE was surgically treated with pars plana vitrectomy and internal limiting membrane inverted flap technique. There was rapid improvement of the anatomical and functional features of the LE macula, the LE VA reaching 6/9.5 at one-month visit.

Conclusion: This is, to our knowledge, the first case of lamellar hole associated with lamellar hole epiretinal membrane (LHEP) surgically treated using the internal limiting membrane inverted flap technique. Further studies are needed in order to better asses the method's anatomical, tomographic and visual outcomes and to establish its indications.

Keywords: Lamellar Macular Hole; Inverted Flap Technique; Epiretinal Membrane; Pars Plana Vitrectomy

\section{Introduction}

Idiopathic lamellar macular hole is a disorder of the vitreoretinal interface, consisting of disruption in the foveal inner retinal layers without extension to the photoreceptors and the retinal pigment epithelium [1]. Two types of epiretinal membranes are commonly associated with lamellar macular holes, namely the conventional ERM, characterized by traction, and atypical degenerative ERM, a dense proliferation, without tractional properties, that appears thicker on the OCT [2]. Surgical treatment is indicated, among other factors, when there is significant loss of visual acuity and/or severe metamorphopsia [3]. The standard technique encompasses pars plana vitrectomy (PPV), ERM removal, followed by ILM peeling, with the most common serious complication: the development of full thickness macular hole (FTMH) [4]. Inspired by the inverted flap surgical technique for FTMHs first described by Michalewska et al. [5], we propose a similar surgical approach for the treatment of one case of LMH, considering the advantage of minimizing the risk of postoperative FTMH.

\section{Methods}

The present interventional report describes the case of a middleaged healthy male patient earlier diagnosed with BE ERM, which exhibited a two-year progression to LMH. Because of LE VA drop, a surgical approach was chosen. The surgery was performed using 
a standard pars plana vitrectomy equipment (CONSTELLATION, Alcon Surgical, Ft. Worth, TX), Topcon OMS-800 OFFISS microscope (Topcon, Japan) and NGENUITY 3D visualization system (Alcon Surgical, Ft. Worth, TX). The surgical technique encompasses standard 25-gauge 3-port pars plana vitrectomy, followed by ERM removal, ILM dye staining (Triamcinolone Acetonide, Vitreal S, BIOSOOFT, Italy) and peeling. The ILM removal was performed in a circular fashion, leaving approximately 1.5 disk diameters of ILM centered on the foveal defect. The ILM flaps were gently handled from all sides until becoming inverted over the LMH. Fluid air exchange was performed, and at the end of the intervention $2 \mathrm{~cm} 3$ C2F6 100\% was injected trough the trocar.

\section{Results}

A 61-year-old patient without any significant past medical history first presented to our service two years ago for an eye consult. At that time, the visual acuity was unaffected, and the patient was diagnosed with BE epiretinal membrane, showing no tomographic signs of retinal layers' disruption. Two years later, the patient started to complain of LE metamorphopsia and VA drop. On the current examination, Snellen's VA was 6/6 in the RE and 6/19 in the LE. The intraocular pressures and the anterior segments were normal in both eyes. Fundoscopy revealed a glisteny membrane on the surface of the retina and a round-elliptical hole with medium depth and no deposits located directly in the center of the macula in both eyes. Spectral-domain optical coherence tomography (OCT) showed morphological changes of the foveal contour with epiretinal membranes, disruption of the inner retinal layers, intraretinal splitting and intraretinal cysts, more advanced in the LE compared to the RE (Figure 1). Due to the patient's symptoms, visual acuity drop and the disorder's anatomical progression, the patient's LE was surgically treated using the internal limiting membrane inverted flap technique, as described before. There was rapid improvement of the anatomical and functional features of the macula (Figure 2), the LE VA reaching 6/9.5 at one-month visit.

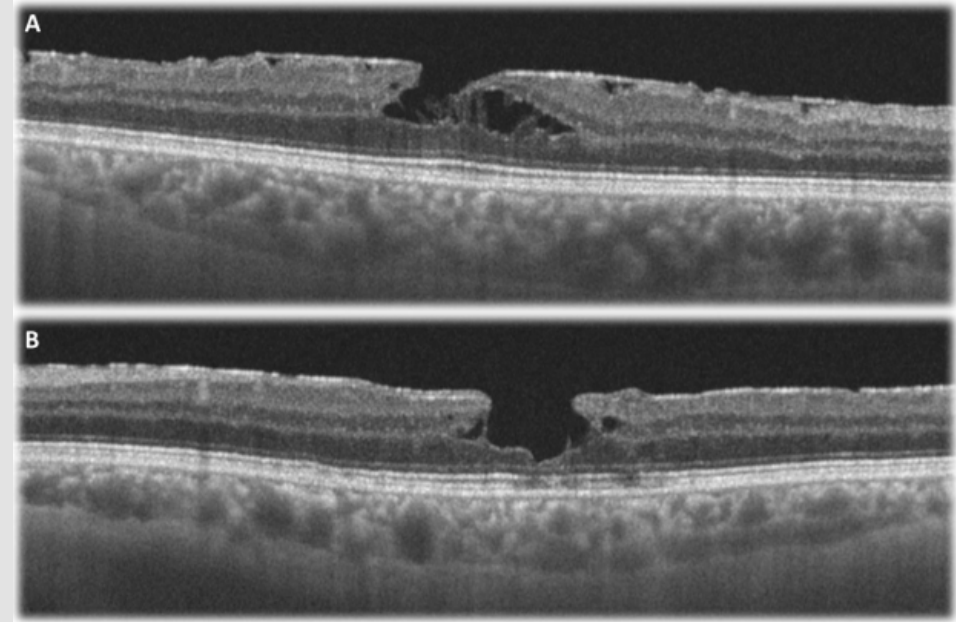

Figure 1: Preoperative macular optical coherence tomography with horizontal scans of the fovea of the

A. Right eye and

B. Left eye.

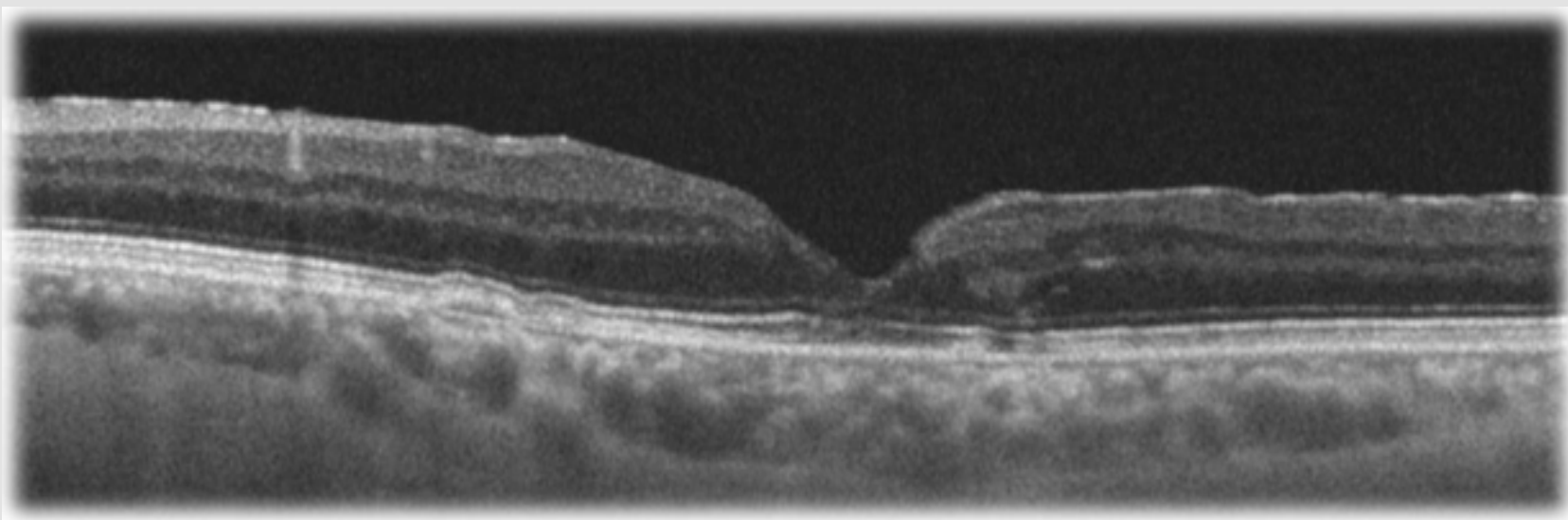

Figure 2: Postoperative macular optical coherence tomography with horizontal scans of the fovea of the left eye. 


\section{Discussion}

There is currently no set agreement that has been reached among the retina specialists on the management of LMHs. The surgical approach or the simple observation are still under debate. The surgical procedure's goal, namely pars plana vitrectomy with ERM and ILM peeling, is to reestablish the foveal profile, by removing the tractional forces and allowing the resolution of the intraretinal edema. Vitreous tamponade (air or gas) at the end of the surgery is also under discussion [6]. We presented the case of a healthy male who developed LMH two years after the diagnosis of ERM in both eyes. This is, to our knowledge, the first case of lamellar hole associated with lamellar hole epiretinal membrane surgically treated using the ILM inverted flap technique, showing significant improvement of the anatomical and functional features of the LE macula at one-month follow-up. There is, however, a new procedure described for LMH associated with atypical ERM, consisting of double inverted ERM and ILM flap technique, which shows better functional prognosis compared to the standard surgical approach [7]. To conclude, further studies are warranted to assess the LMH surgical techniques' anatomical and functional outcomes in order to establish the correct management of these patients

\section{ISSN: 2574-1241}

DOI: 10.26717/BJSTR.2019.23.003892

Ioana Teodora Tofolean. Biomed J Sci \& Tech Res

(C) This work is licensed under Creative Submission Link: https://biomedres.us/submit-manuscript.php

\section{References}

1. Witkin AJ, Ko TH, Fujimoto JG, Schuman JS, Baumal CR, et al. (2006) Redefining lamellar holes and the vitreomacular interface: an ultrahighresolution optical coherence tomography study. Ophthalmology 113(3): 388-397.

2. Govetto A, Dacquay Y, Farajzadeh M, Platner E, Hirabayashi K, et al. (2016) Lamellar macular hole: two distinct clinical entities? Am J Ophthalmol 164: 99-109.

3. Haritoglou C, Schumann RG (2017) Indications and surgical approach for lamellar macular holes and pseudoholes. Ophthalmologe 114(12): 1117-1121.

4. Witkin AJ, Castro LC, Reichel E, Rogers AH, Baumal CR, et al. (2010) Anatomic and visual outcomes of vitrectomy for lamellar macular holes. Ophthalmic Surg Lasers Imaging 41(4): 418-424.

5. Michalewska Z, Michalewski J, Adelman RA, Nawrocki J (2010) Inverted internal limiting membrane flap technique for large macular holes. Ophthalmology 117(10): 2018-2025.

6. Hirakawa M, Uemura A, Nakano T, Sakamoto T (2005) Pars plana vitrectomy with gas tamponade for lamellar macular holes. Am J Ophthalmol 140(6): 1154-1155.

7. Frisina R, Parrozzani R, Pilotto E, Midena E (2019) A Double Inverted Flap Surgical Technique for the Treatment of Idiopathic Lamellar Macular Hole Associated with Atypical Epiretinal Membrane. Ophthalmologica 242(1): 49-58.

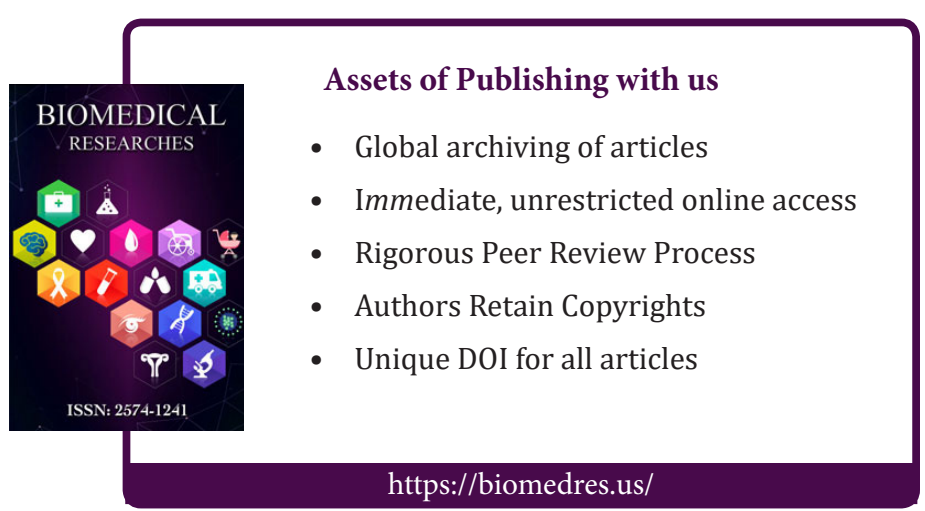

